\title{
Fundamentals of systems ergonomics
}

\author{
John R Wilson \\ Human Factors Research Group, Faculty of Engineering, University of Nottingham, Nottingham NG7 2RD, UK
}

\begin{abstract}
Ergonomics/human factors is, above anything else, a systems discipline applying a systems philosophy and systems approaches. Many things are labeled as "systems X" in today's world, and this paper specifies just what attributes and notions define ergonomics/human factors in systems terms. These are concern for context, acknowledgement of interactions and complexity, a holistic approach, recognition of emergence and embedding of the professional effort involved within organization systems. These five notions are illustrated with examples from a large body of work on rail human factors.
\end{abstract}

Keywords: Systems, systems ergonomics, human factors, rail

\section{Introduction}

It has long been argued that, to be meaningful, ergonomics/human factors (E/HF) is a systemsoriented discipline. Many of the early experts and authors in ergonomics/human factors (eg Chapanis, Corlett, Singleton) saw it clearly as a systems discipline (eg Singleton, 1974 in a short but prescient book). Several leading authorities (Sheridan, Rouse, Rasmussen etc) actually came into E/HF from a systems engineering background, and worked with many people from control engineering who brought systems-level models with them. Their interest in human capability and fallibility, when it became clear that apparently advanced process control systems would fail if they induced errors in operators and managers (and, following Bainbridge, actually needed human expertise to work properly), spawned a movement in cognitive systems engineering and subsequently joint cognitive systems (Hollnagel, Woods). It is not surprising that there is a systems design orientation to the work of those mentioned above, and many others in $\mathrm{E} / \mathrm{HF}$, since they are usually dealing with large and complex systems, with many interacting components. However, even within an ergonomics applied to industrial workplaces and physical work, leading ergonomists worldwide have clearly seen that we can only usefully address the relevant human factors concerns at a systems level, whether we call it systems ergonomics, or participatory ergonomics/design or, as has become prevalent in north America at least, macroergonomics.
However, in parallel with all this support for the primacy, indeed necessity, of a systems view, in some areas of ergonomics application it sometimes seems that a single problem-single solution ethos still prevails. This may be for a number of reasons. Most acceptably, it may be because of the impracticality in some settings, or given some project or investigation remits, to do more than concentrate upon a micro view of the human factors involved, to say "yes I know there are larger systems issues afoot but I only have time/permission/access to address this small part of the problem". Less acceptable are those cases where a narrow non-systems approach is taken because the investigators concerned are only competent in or interested in a narrow channel of E/HF; the manual handling charlatans of a few years ago come to mind.

Rather than make a plea for a systems-oriented ergonomics it is tempting to be hard-nosed and suggest that any study, investigation, analysis or development which does not take a systems view is, in fact, not ergonomics at all. Rather such an initiative is seen as a biomechanical, cognitive psychology or physiology study, and of limited practical value. So, a musculoskeletal disorders investigation or improvement which does not account for psychological/emotional/social influences, on MSD causation or success of solutions, is not fully ergonomics; assumptions that cognitive task performance occurs in a vacuum away from emotional influences and motivational environmental impacts means that any findings have less value; treating people as isolated indi- 
viduals and as "lonely end users" (Nardi, 1993) is not a sensible route to developing better interfaces or jobs. Such a position might be too extreme for some, but it does start to more clearly delineate what is our discipline and what is not.

Why this paper at this time? Well, if the world of $\mathrm{E} / \mathrm{HF}$ is to have a future then we have to accept that it is a systems discipline and that, to paraphrase Hal Hendrick, good ergonomics is systems ergonomics. However, it is all very well to espouse the systems viewpoint and approach. We need to be clearer what we mean. This paper brings together ideas from earlier efforts (eg Moray, 2000, Wilson, 2000), other sources from outside our discipline, and practical experience in different industries to move beyond the easy statement of ergonomics as a systems discipline and to try to exemplify what we mean by this.

\section{Systems engineering and systems ergonomics}

So, what is systems ergonomics? An easy answer, if a somewhat circular one, is that systems ergonomics examines, accounts for and enhances the design of a system, rather than of an individual component part, whether artefact, facility or person. Which immediately begs the question of: what is a system? If we agree that the notion of systems ergonomics is key to the ergonomics/human factors profession, then we need to understand what is agreed, or not, on the systems and of systems engineering.

Chapanis (1996) notes that "systems" is used in many ways but he concentrates on what he calls "equipment systems", which he defines as "an interacting combination, at any level of complexity, of people, materials, tools, machines, software, facilities, and procedures designed to work together for some common purpose." (p20). I think most of us today would agree with a definition something like this, except that we would move away from the "equipment" tag and the idea that systems are collections of hardware, software, buildings and people, and add some notion of social systems, communities and collaboration. Given some current debates over the term "human system integration" it is interesting to note that Chapanis does refer to a debate even back then over whether the person should be conceptualised as inside or outside the system, and he points out that all non-natural systems ultimately have some human purpose and have some interfaces with people.

Any understanding of systems ergonomics must be related to systems engineering. And it is here that we have another problem because of the variety of view- points and opinions available - not an unusual situation! In a recent initiative at my own university, views on systems engineering varied from those who saw it purely as the modern approach to electronic and electrical systems design (I am being slightly unfair here) to those of us who not only saw it as having concern for people at its core but who believe that there is no systems engineering without central concern for human factors. Where we were in agreement was that it is impossible to understand the problems facing design, planning and organisation required to deliver effective, safe, reliable, efficient systems without taking a systems perspective.

In some ways the view of Chapanis (1996) is close to my own. His selection of definitions of systems engineering veers towards ones which, with small changes, might also define a design-oriented human factors. He also suggests that debate over the nature of systems engineering is not settled, but builds his definition around the ideas of understanding (evolving) user needs, incremental development of requirements and specifications, and that systems engineering includes integration of all disciplines throughout the system life cycle so as to assure that all user requirements are satisfied.

The antithesis of a systems approach to development was seen clearly in a recent proposal for a major international project reviewed and evaluated by the author. In this, some very clever use of future mobile and ubiquitous technology and wireless networks was proposed, in order to create a citizen participant movement for sensing environmental traces and communicating these to centralised databases and knowledge management systems. The technology looked both advanced and feasible. But, no mention was made of issues such as motivation, privacy, acceptability to local government, unintended consequences of people using the information for commercial purposes etc. OK, so they had forgotten, or just not recognised, the human factors issues, and we are used to that from (some) systems developers. But they also made no mention of equipment robustness, maintainability, battery life, replacement policy, spares availability, integration with other local and national community systems, and so on. In other words, there appeared to be little or no systems thinking within the proposal.

\section{Notions in systems ergonomics}

In my view there are six overlapping defining features of systems ergonomics - system focus, context, 
interactions (including complexity), holism, emergence and embedding.

\subsection{Systems focus}

The first feature may seem blindingly obvious, indeed even trivial, but needs stating all the same. That is systems ergonomics treats the focus of interest as a system - as an interacting combination of DNA, materials (organic and inorganic), bytes, functions/processes and ideas. This combination may not be stable, indeed where systems are complex it they will not be, and our focus is on the inter-connections as much or more than the components. Our systems of interest used to be defined as human- (or man-) made at one time, but I think now that most systems ergonomists would say that we also have an interest in the investigation and "design" of people's interaction with or within natural systems if not design of the natural systems themselves.

\subsection{Context}

Moray (1994) amongst many others proposed that all behaviour and performance takes place in a setting or a context, and ergonomics must understand and account for this context, which increasingly is that of complex socio-technical (or just social) system. The extension of this argument is that most E/HF should take place in field settings (see de Keyser, 1992, de Montmollin and Bainbridge, 1985), and that the problem with all our laboratory work is that even the most statistically significant of results in the best controlled experiment only account for a fraction of the real variance in real practice (largely due to the issue of complexity as alluded to above). An extension of this view is to suggest that few if any laboratory studies are properly ergonomics because they cannot account properly for the complexity and multiple factors found in real settings. My own view is that systems ergonomics should be carried out "in the wild" except for where ethical, substantial pragmatic or sheer unavailability issues intervene.

If everything that people do and everything that E/HF studies, improves and implements, takes place in a context, and is part of a system of goals, activities, means and artefacts, then we must define what within the system of interest is of proper interest to E/HF and to our study, and what lies outside this even though impacting on the work we do and the systems we critique or design. If we are working at a systems level then in defining our system we need some version of the old task analysis stopping rule, but working outwards perhaps even more than inwards. In other words, where is the boundary of our system of interest, at what point does our system end and another - companion or child or parent system begin? Deciding on this, and being clear about it, is the first step in any analysis for a systems ergonomics initiative. There are no real rules for doing this other than being clear and being sensible in terms of acknowledging that the effort must produce something useful, and recognising that the boundary for one form of the system may be different than that for another. For example, if our study is of behaviour of process control room operators with a view to the design of better information interfaces, then we could be interested in the position of electrical power points (for layouts to support high quality work behaviour) but certainly not how the national electricity grid works; we may be interested in the design of operating procedures and their influence on violations but in this case also need to know something about the working of the national regulator; we may have a central issue the choice between large shared screens and personalised displays but, at the level of performance we are concerned with, not concerned about the font size and contrast on them.

\subsection{Interactions}

The basic nature of a "system" is that it consists of interacting parts. Or at least that is how a systems approach typifies the object of interest. This very fundamental view lies at the heart of many of our approaches and concepts - human-machine systems, socio-technical systems, joint cognitive systems etc. At the heart of ergonomics lies the notion of a system of interacting components, made up of materials (organic and inorganic), bytes, DNA and ideas, and that it is the integration of these components which lies at the heart of good E/HF. Our purpose is the integration of human, technical, information, social, political, economic and organisational components within the system and optimisation (or at least satisfise) of the interactions involved. The purpose of E/HF is to integrate as seamlessly as possible these different human and technical components. Such integration is principally within the development and implementation process, but continues into operational use, maintenance, testing and decommissioning. $\mathrm{E} / \mathrm{HF}$ is about the understanding and design for the interactions not the components; we design, say, a "person/people - building" interaction not a building, that 
we analyse a "person-computing device-person" interaction or even a "person-device-device-person" interactive network rather than an interface or device alone. In our world of $\mathrm{HF} / \mathrm{E}$ as well the interactions we are interested in are purposive and purposeful.

To take a systems ergonomics approach means that we do not treat equipment or workplace or wider system development as if it were an island, separate from the end users and other stakeholders. As parallels: production organizations must account for supply chain interactions and influences, on local and international scales; transport operators must account for the inter-operability of their operations with those of other modes of transport or even countries; software engineering enterprises will integrate planning for development across the product life-cycle and will allow for integration with other softwares; procurement groups will account for the interfacing of new pieces of equipment (physically or in software) with existing systems; and manufacturers will examine the design and implementation of a production system with respect to its interaction with maintenance systems in a design for maintainability approach.

This argument, of the most useful E/HF having a focus on the interactions themselves and not the things interacting, is basic to many approaches within E/HF (Wilson, 2000), such as joint cognitive systems for person-artefact interactions, or distributed cognition for people - people-work tasks - organisations.

Related to the notion of interaction is the idea of complexity. It is the very complexity of the world that is part of the rationale for a formal discipline and profession of E/HF. Wisner (1994) talked of the inapplicability of the linear model as the paradigm for ergonomics in the context of recognizing the effects of complexity. This complexity, and the sheer variability of people (massively increased when many people are interacting together), leads us to another facet of the integrated nature of systems, uncertainty

\subsection{Holism}

The discipline and profession of $\mathrm{E} / \mathrm{HF}$ is holistic (or should be); that is we have concern for and seek to understand physical, cognitive, and social (and increasingly today emotional) characteristics of people in order to enhance the interactions they have with artefacts, information, environments and other people. E/HF must see the human in the round, or holistically - physically, mentally, socially and emotionally. Such a perspective is its very raison d'être. For simplicity of explanation to the outside world and to rationalise the broad church of ergonomics, we may describe various technical branches of ergonomics - at a top level physical, cognitive, organisational, and at a level below forensic, macro, participatory etc. But whilst this is understandable it is potentially dangerous if it leads us to think that the discipline can be so easily partitioned. In a holistic systems view the cognitive, physical and social must combined to the extent which is appropriate within the study, investigation, analysis, and solutions that we work with.

The above discussion has concerned the holism of E/HF inputs, but outputs should be addressed holistically also. This is in terms of concern for the impact of people on the performance of the human-machine, socio-technical and social systems, and the impact of system design on the well-being of all stakeholders (ie all effected by or coming into contact with the system) - physically, mentally, emotionally.

\subsection{Emergence}

The fourth significant feature of a systems ergonomics approach is the recognition of the emergent properties of systems, including the emergent properties of the human components.

I see emergence as central to systems ergonomics in three ways. First, all systems in real use, with real users and under the constraints of time, space, motivation etc found in practice, will display characteristics and operate in ways not expected or planned for by their designers. Every ergonomist working with real companies will be familiar with the cry from the engineers - "but we didn't expect them to do that!" The history of failure of grandiose ICT projects is littered with examples of end users who did not (and never had if the developers had troubled to find out) behave as the job specification, operating procedures or managers' idealisations suggested. Dysfunctional emergent properties of the human-machine system can be reduced through better human factors integration throughout the development lifecycle.

The second type of emergent properties is intimately related to the first, whereby the impact of poor designs may be mitigated through the well recognised ability of users to find a way to make the system work despite its shortcomings. As recognized by Lisanne Bainbridge's ironies of automation many years ago, people find ways to accommodate, avoid, overcome or work around system deficiencies which were predicated on poor intelligence about the context, the tasks, the constraints, the users and their needs and contributions. Thus people may behave in 
non-specified and non-predicted (though probably predictable) ways .beneficial to system performance; ways of working with a system emerge due to the ingenuity of people.

Third, and again positively, there is emergence where people unexpectedly take advantage of capabilities not dreamed of by designers or find new system uses. We are all familiar with the child who finds pleasure in using the box for imaginative games rather than the toy inside it, and the well known example of the possibility of widespread texting being little thought of by early mobile phone developers, but this kind of emergence can be found in many situations. Broadly there is the increased inroads into large company use of COTS (commercial off the shelf technologies) and the way capital equipment finds its way onto the consumer market.

The E/HF practitioner, whilst not blessed with $20 / 20$ predictive capability should at least be alert to changes in use of products and systems, both dysfunctional and functional. E/HF analyses and evaluations should allow for the possibility of emergence from the inter-twined economic, political and other interactions as well as the physical, cognitive and social ones we may have designed for.

\subsection{Embedding}

The sixth feature of a systems approach is the way in which we as ergonomists carry out our work, and who we do this with. This means the way ergonomics fits within the organisational system and is embedded within practice. Of course good ergonomics is participatory and so, as far as possible, we work with all key job holders and subject matter experts. However, all large companies with an ergonomics/human factors function have to make a choice - where to place them within the organisation. This might be within operations, design, engineering, safety, training and so on. Some companies might try to distribute human factors across all these groups and more, but unless their numbers are large this, in my experience, runs the risk of such small (sometimes single person) groups being isolated, being picked off or marginalised, or even them going native and ceasing to offer an ergonomics view separate to their host department.

\section{Rail systems ergonomics}

To give practical examples of systems ergonomics I could take any of a number of projects from the work between University of Nottingham and Net- work Rail. This programme has included fundamental research to develop new ideas, theory, tools and knowledge, and applied research to improve rail work systems. Figure 1 provides a simple model of our contribution to the wider rail systems engineering work, capturing the holistic nature of $\mathrm{E} / \mathrm{HF}$ and the contribution to people and human-machine systems performance, the equipment and interfaces they use and the wider systems and organisations involved. We carry out or support analysis, design and integration activities, in order to understand people's knowledge and competence (who we are), their tasks and functions (what we do), the artefacts to support their work (how we do it) and the setting, culture and context they perform in (where we do it).

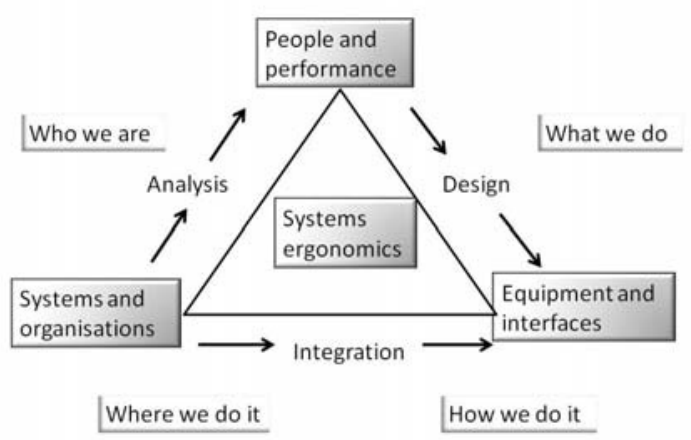

Figure 1: Model of systems ergonomics

\subsection{Rail systems ergonomics}

Without wishing to overstate the case there has been something of a shift in the approach of human factors in domains such as rail over the past few years, with human factors taking a holistic, sociotechnical and root cause view on systems. Furthermore, the human roles, functions and activities are often distributed - temporarily, spatially and functionally. Activity in a system may take place continuously or discretely over a period of time and we can "follow" the artefacts or people of interest through that system over time (an equivalent here is following the "patient journey" through a health system - eg Buckle et al, 2010)). Action may also takes place over a wide geographical area, for instance the work of a forest fire fighting crew as they carry out their work in a command centre, on the ground in the forest and from the air. Activity may also be functionally distributed, for instance an Olympics design 
and development team containing planners, concept designers, engineers, architects, stylists, production engineers, systems engineers, marketing specialists and customer representatives.

We can see this trend to take a systems viewpoint when studying rail in a number of instances. For instance early work to examine how to support passengers through architectural design or information display carried out classical studies of design of signs, placement in key positions within stations etc. Most recently such studies have tracked passenger journeys, from leaving home or at least arrival at the station, through all aspects of access, information finding and train boarding, and then subsequently alighting at the other end, and include interaction with other forms of transport in a multi-modal view. The running example used in the rest of this paper is our research into track maintenance and engineering activities, including access and protections, which investigated the roles and contribution of a number of functions in rail maintenance such as the person in charge of possessions, engineering supervisors and controllers of site safety (Wilson et al, 2009).

\subsection{Context of the rail network and rail companies}

As far as context is concerned, this is critical for the railway. At the top level government policy and strategies, especially in terms of the organisation of the industry (how it is divided up between infrastructure owners and rail operating companies for instance) and the commercial and operating contracts available, has a profound effect on meeting human factors requirements. Strongly related to this, the position of the railways in the public's affection (in many societies) effects their perception of when things go right or wrong, including their perception of how much safety they can expect (as with other complex systems such as healthcare, they perhaps expect greater levels of safety than can possibly be delivered). At another level we have the regulators, those concerned with health and safety as are found in any safety critical industry, and those concerned with commercial contracts and public value for money. Then, and the astute reader will realise that we are following Rasmussen's (1997) stages of risk management in large systems, there are the layers of the organisation, its management, its supervisors, all direct and indirect staff, and the hardware and software systems they work with. Whilst it would be idle to pretend that in every piece of work we do on the railway we account explicitly for all these levels, we do so wherever possible. For instance our work on the understanding of planning for engineering work was strongly related to the impact of the regulator, and of intra- and inter-organisation levels; our function analysis leading to risk analysis was clear about the interactions between technical supervisory, safety supervisory, planning and track workers levels.

\subsection{The railway is a distributed interactive system}

In relation to interactions, in essence the railway is a large, complex distributed system of many technical, organisational, economic and human components. This distributed system is spread across regional, national and cultural boundaries, giving additional problems of inter-operability. Clearly this is a system of inert-connected parts, with multiple links, and complex in functional, topographic, temporal and communication terms. The links are multiple and constantly changing.

To continue with our earlier example, from an ergonomics perspective all aspects of rail engineering and the rail system are interconnected and a number of efforts have been made to typify the system involved, to establish how different parts interact, from planning through to delivery, and including how both safety as well as efficient performance is propagated through the system (Ferreira, 2011. White, 2011). Amongst the routes used to do this we have included social network and communications analyses, command/control graphics, models and metrics of resilience etc.

Human factors can only be successfully accounted for, and an ergonomics function can only add value, if an integrated systems view is taken of activities and processes throughout the development and operational life of any system. In the railway this involves an integration plan with consideration of all operational modes (normal, abnormal, degraded and emergency), across technical (for example, lineside signals and in-cab information displays) and organisational (train and freight operating companies, projects and regions) systems.

An extension of the inter-connectedness and complexity of the systems that we deal with is into the fact that all such systems will have multiple goals, and frequently competing goals. This is clearly seen in the trade-offs that must be made across our ergonomics goals (or criteria) of safety, reliability, efficiency, use of capacity, security, environmental sustainability and so on (Wilson et al, 2009). 


\subsection{Holistic approaches to railway ergonomics}

The rail network is an excellent example of a complex sociotechnical system - the health service, emergency services, off-shore gas and oil are others where every kind of work and human factor is present, and therefore the idea of ergonomics being holistic is vital. The stakeholders include signallers and controllers (electrical, infrastructure fault and traffic); drivers; station and on-train staff; planners, engineers and managers; track (maintenance) engineers and workers, lookouts and site safety controllers; passengers and the general public (the last both legitimate - eg at level crossings, and illegitimate eg trespassers). The rail system includes work in vehicle control, systems process control, monitoring, planning and physical work, occurring in settings such as vehicle cabs, control rooms, outdoors in all weathers, and large buildings and spaces. The artefacts used include VDUs, signals, paper, CCTV, hard wired controls, handtools and large engineering plant and vehicles. Therefore the human factors contribution must be multiple, combining cognitive, physical and social theories, methods and knowledge.

Linking the contextual and the holistic in our approach to systems ergonomics, in the rail engineering work of the running example in this paper we have examined the use of mobile computing (Dadashi $\mathrm{Y}$ et al, 2011a; Dadashi, 2008). Clearly use of such devices involves examination of physical, cognitive and organisational interactions, and the way these are multiply connected. The devices need to be usable in all outdoor conditions, robust yet easy to handle; the screens need to be able to present location maps and access routes, maintenance histories, technical advice and to do so in a meaningful and accessible manner given space constraints; and we need to understand the change in organisational relationships and communications links once more information access and thereby control is given in the field to "remote agents". To allow for context we developed a functional use assessment tool, EDARE, to understand what different functional needs, and thereby information access requirements, different job groups would have in the practice (Dadashi et al, 2007). This has been extended more recently into similar analyses of information needs for different functional groups as contemporary intelligent infrastructure systems begin to be implemented (Dadashi $\mathrm{N}$ et al, 2011b).

\subsection{Emergent properties of rail systems}

Two particular facets of emergence are relevant to our work in rail engineering, both implicit in the discussion of holism. First of all, the advent of intelligent infrastructure will have a profound effect on how things are done (the aim is a move from find and fix to predict and prevent) and who does those things (Dadashi et al, 2011a). Key decisions will have to be made about the degree to which the continual monitoring and diagnosis of the state and performance of assets is handled by people or automated systems, and how these will be combined, the extent to which first and second level alerting and decision making will take place on-site (with potential benefits of immediacy, local knowledge and location appropriate decisions and actions) or remotely and centrally (with potential benefits for cost, consistency and colocation of key expertise); and how to reduce what will be the billions of bits of data possible, down to manageable knowledge and intelligence to be used by people with different agendas - that is development of good data screening/filtering, and conversion into intelligence via human-centred and ecologically valid displays.

The second facet of emergence is shown when we study how people's jobs will change as their information sources and decision supports become more mobile and personal (Dadashi et al, 2011b), and as information becomes ambient. We will see the emergence of new roles, communication channels, relationships, power structures, sources of decision making and liaison/collaborations.

\subsection{Embedding ergonomics/human factors in the rail industry}

As regards embedding, in our own case of the team within Network Rail we have always been based within the engineering function. Given what was said earlier about systems ergonomics and systems engineering this is seen as the best place for us to be. Certainly we have had greatest possible impact on new systems and projects, and on maintenance, renewal and enhancement activities, through this positioning, and via the strategic relationships we have set up with operations as well. The point is that wherever the ergonomics function is sited it must collaborate with all other functions to do its job. In fact, putting it stronger, it is often because a good systems-oriented ergonomist actually does work with all other functions, indeed has to in order to do their 
job, and must understand all stakeholder needs (which includes those of others managing or building the system as well as of end users) that ergonomists are so valuable to a successful systems engineering.

\section{Conclusions}

The debate over systems ergonomics has become more urgent as we seek to maintain and strengthen ergonomics/human factors as a discipline and a profession. There might seem to be some irony in ergonomics best addressing the worlds it investigates at a systems level, since systems implies dealing with a number of constituent parts rather than being holistic. But, as many systems thinkers point out, we should better see the system as a whole, as holistic (eg Angyal, 1969). The corollary to this argument is that any ergonomics which is not systems in orientation is of less value, may even be in danger of providing poor solutions to problems of people's interaction with things, ideas and settings.

Of course it is rare that any one ergonomics study, initiative or even programme can embrace every aspect of the system and hence human factors. Resource availability and indeed logic dictate against this In the author's own work there have been times when the enormity of trying to address and in particular analyse all aspect of a system became too much and we concentrated on particular system parts instead (eg Wilson and Whittington, 2001). But even then, the rest of the wider system was acknowledged as context.

Many years ago Nigel Corlett said that "[a systems view] does not mean dumping the linear concept, many of our problems can be solved via a linear paradigm and for many situations it is a powerful approach. But it is not enough .... and we must take on board some of the more recent [systems] thinking if we are to keep up with need." (Corlett, unpublished paper to the IEA Exectutive, 1996). Even in projects which have been set up to have a narrow focus, perhaps because of sponsor and client constraints, there is a need to know what the wider system is, what the context and what the key interactions, and how all this can be accounted for in the interpretation and use of findings even if not completely in their generation.

\section{References}

[1] A. Augyal, A logic of systems, in: Systems Thinking, F.E. Emery, ed., Penguin Books, 1969.
[2] P. Buckle, P.J. Clarkson, R. Coleman, J. Bound, J. Ward and J. Brown, Systems mapping workshops and their role in understanding medication errors in healthcare, Applied Ergonomics 41 (2010), 645-656.

[3] A. Chapanis, Human Factors in Systems Engineering, John Wiley \& Sons, 1996.

[4] Y. Dadashi, J.R. Wilson, S. Sharples and T. Clarke, Applications of handheld computers for the rail industry, in: Contemporary Ergonomics 2008 Proceedings of the Ergonomics Society Annual Conference, Nottingham, P.Bust, ed., Taylor and Francis, London, 2008, 546-551.

[5] Y. Dadashi, J.R. Wilson, S. Sharples, D. Golightly and T. Clarke, First model of human factors in intelligent infrastructure systems, Proceedings of Ergonomics and Human Factors Annual Conference, 2011, pp.147-154.

[6] Y. Dadashi, S. Sharples, J.R. Wilson and T. Clarke, accepted for publication in Journal of Personal and Ubiquitous Computing, 2011.

[7] V. de Keyser, Why field studies? in: Design for manufacturability: A systems approach to concurrent engineering and ergonomics, M. Helander and M. Nagamachi, eds., Taylor \& Francis, London, 1992, pp. 305-316.

[8] M. de Montmollin and L. Bainbridge, Ergonomics and human factors? Human Factors Society Bulletin 28 (6) (1985), 1-3.

[9] T. Farrington-Darby and J.R. Wilson, Understanding social interactions in complex work: a Visual Ethnography, Cognition Technology and Work, 11 (2009), 1.-15.

[10]N. Moray, "De Maximis non Curat Lex" or how context reduces science to art in the practice of human factors. Proceedings of the Human Factors and Ergonomics Society $38^{\text {th }}$ Annual Meeting, Santa Monica,, Human Factors and Ergonomics Society, CA, 1994, pp 526-530.

[11]N. Moray, Culture politics and ergonomics, Ergonomics, 43 (2000), 858-868.

[12]H. Patel, M. Pettitt, and J.R. Wilson, J.R., Factors of collaborative working: A framework for a collaboration model. Applied Ergonomics, 43(2012), 1-26.

[13]J. Rasmussen, Risk management in a dynamic society: A modelling problem, Safety Science 27 (1997), 183-213.

[14]A. Schock, B. Ryan, J.R. Wilson and T. Clarke, Visual scenario analysis: Understanding planning in rail engineering, Journal of Production Planning and Control 21 (2010), 386-398.

[15] W.T. Singleton, Man-Machine Systems, Penguin Education, 1974.

[16]J.R. Wilson, B.J. Norris, T. Clarke and A. Mills, eds; Rail Human Factors: Supporting the Integrated Railway, Ashgate, London, 2005.

[17]J.R. Wilson, B.J. Norris, T. Clarke and A. Mills, People and Rail Systems: Human Factors at the Heart of the Railway, Ashgate Publishing, Abingdon, UK, 2007.

[18]J.R. Wilson, T. Farrington-Darby, G. Cox, R. Bye and G.R.J. Hockey, The railway as a socio-technical system: Human factors at the heart of successful rail engineering. Proc. IMechE, 221, Part F, Journal of Rail and Rapid Transit (2007) 101-115.

[19]J.R. Wilson, B. Ryan, A. Schock and J. Pitsopoulos, Understanding safety and production risks in rail engineering planning and protection, Ergonomics 52 (2009), 774-790.

[20] J.R. Wilson, Fundamentals of ergonomics, Applied Ergonomics 31 (2000), 557-567.

[21] J.R. Wilson and C. Whittington, Implementation of self managed teams in manufacturing: more of a marathon than a sprint, AI and Society 15 (2001), 58-81. 